\title{
Modelling the temperature conditions in three-dimensional piecewise homogeneous elements for microelectronic devices
}

\author{
V.I. Gavrysh \\ National University “Lviv Polytechnic”, 28-a, S. Bandery str., 79013 Lviv, Ukraine \\ E-mail: ikni.pz@gmail.com
}

\begin{abstract}
The steady-state linear thermal conductivity problem for an isotropic layer with a thin foreign parallelepipedic inclusion that releases heat has been considered with account of heat dissipation. The methodology for analytic solution of three-dimensional steady-state boundary thermal conductivity problem has been suggested.
\end{abstract}

Keywords: temperature, thermal conductivity, steady-state, isotropic, heat dissipation, ideal thermal contact, foreign inclusion.

Manuscript received 19.05.11; revised manuscript received 15.08.11; accepted for publication 14.09.11; published online 30.11.11.

\section{Introduction}

The investigation of heat conductivity in the elements of microelectronic devices contributes to better understanding of a number of microprocesses that take place in them and are related to the movement and dissipation of elementary particles. None of the transfer phenomena provides such rich and deep information about lattice vibrations, about the excited states of electrons, about the interaction of electrons with lattice in semiconductors, as thermal conductivity does. In microelectronic device elements, where electric current flows, nonhomogeneous heat release always takes place, and in some cases large temperature gradients arise. Therefore, the performance of microelectronic devices strongly depends on the temperature regimes imposed during heating process. This is especially significant in devices, where the thin-film layers and the foreign inclusion are applied.

Some researches of the temperature regimes for separate elements of microelectronic devices have been conducted earlier [1-4].

Hereinafter the boundary steady-state thermal conductivity problem has been formulated, the analytical solution and the numerical analysis for the element of microelectronic device, which is described by an isotropic layer with thin parallelepipedic inclusion that releases heat and by heat dissipation. The general thermal conductivity equation for piecewise homogeneous structures has been presented $[5,6]$.

\section{Problem statement}

Consider the isotropic layer containing parallelepipedic inclusion with the volume $V_{0}=8 \mathrm{hbd}$, in which the area $\Omega_{0}=\{(x, y, z):|x| \leq h, y \leq b,|z| \leq d\} \quad$ of uniformly distributed internal heat sources with the power $q_{0}$ acts. The body under consideration is referred to rectangular Cartesian coordinate system $(O x y z)$ with its origin in the center of inclusion. At the inclusion boundary surface that releases heat, the conditions of ideal thermal contact are fulfilled, and on the layer boundary surfaces $K_{v}=\left\{\left(x, y, d+l_{v}\right):|x|<\infty,|y|<\infty\right\}$, $K_{n}=\left\{\left(x, y,-d-l_{n}\right):|x|<\infty,|y|<\infty\right\}$ the conditions of convective heat exchange with the environment with constant temperature $t_{c}$ (Fig. 1) are given.

\section{Mathematical model of the problem}

Assume that the foreign inclusion is thin. To determine the stationary temperature field $t(x, y, z)$ in the considered system, we use the heat conductivity equation $[3,4]$

$\operatorname{div}[\lambda(x, y, z) \cdot \operatorname{grad} \theta]=-Q(x, y, z)$,

where

$\lambda(x, y, z)=\lambda_{1}+\Lambda_{0} \cdot N(z, d) \cdot \delta(x, y)-$ 
is the thermal conductivity coefficient of the nonhomogeneous layer; $\Lambda_{0}=4 h b \cdot \lambda_{0}-$ reduced thermal conductivity coefficient of the inclusion; $\lambda_{0}, \lambda_{1}-$ thermal conductivities of the inclusion and the layer materials, respectively; $\theta=t-t_{c} ; N(z, d)=S_{-}(z+d)-S_{+}(z-d) ; \delta(x, y)-$ Dirac delta function; $Q(x, y, z)=Q_{0} \cdot N(z, d) \cdot \delta(x, y)$; $Q_{0}=4 h b \cdot q_{0}-$ reduced capacity of internal heat sources;

$S_{ \pm}(\zeta)=\left\{\begin{array}{lll}1, & \zeta>0, & \\ 0.5 \mp 0.5, & \zeta=0, & -\quad \text { asymmetric } \quad \text { unit } \\ 0, & \zeta<0 .\end{array}\right.$ function [7].

The boundary conditions will have the appearance $\left.\lambda_{1} \frac{\partial \theta}{\partial z}\right|_{z=d+l_{v}}=-\left.\alpha_{v} \theta\right|_{z=d+l_{v}},\left.\quad \lambda_{1} \frac{\partial \theta}{\partial z}\right|_{z=-d-l_{n}}=\left.\alpha_{n} \theta\right|_{z=-d-l_{n}}$, $\left.\theta\right|_{|x| \rightarrow \infty}=\left.\theta\right|_{|y| \rightarrow \infty}=0,\left.\quad \frac{\partial \theta}{\partial x}\right|_{|x| \rightarrow \infty}=\left.\frac{\partial \theta}{\partial y}\right|_{|y| \rightarrow \infty}=0$.

Here $\alpha_{v}, \alpha_{n}$ - heat dissipation coefficients of the edges $K_{v}, K_{n}$ of the layer, respectively.

Let us introduce the function

$T=\lambda(x, y, z) \cdot \theta$

and differentiate it with respect to variables $x, y, z$ taking into account description of the thermal conductivity coefficient $\lambda(x, y, z)$ (2). As a result, we obtain:

$$
\begin{aligned}
& \lambda(x, y, z) \frac{\partial \theta}{\partial x}=\frac{\partial T}{\partial x}-\Lambda_{0} \theta(0,0, z) \cdot N(z, d) \cdot \delta_{x}^{\prime}(x, y), \\
& \lambda(x, y, z) \frac{\partial \theta}{\partial y}=\frac{\partial T}{\partial y}-\Lambda_{0} \theta(0,0, z) \cdot N(z, d) \cdot \delta_{y}^{\prime}(x, y), \\
& \lambda(x, y, z) \frac{\partial \theta}{\partial z}=\frac{\partial T}{\partial z}-\Lambda_{0}\left[\theta(0,0,-d) \cdot \delta_{-}(z+d)-\right. \\
& \left.-\theta(0,0, d) \cdot \delta_{+}(z-d)\right] \cdot \delta(x, y) .
\end{aligned}
$$

By substituting the expressions (5) to the relation (1), we come to the differential equation with partial derivatives with discontinuous and singular coefficients:

$$
\begin{aligned}
& \Delta T=\Lambda_{0}\left\{\theta(0,0, z) \cdot \mid \delta_{x}^{\prime \prime}(x, y)+\delta_{y}^{\prime \prime}(x, y)\right] \cdot N(z, d)+ \\
& \left.+\left[\theta(0,0,-d) \cdot \delta_{-}^{\prime}(z+d)-\theta(0,0, d) \cdot \delta_{+}^{\prime}(z-d)\right] \cdot \delta(x, y)\right\}- \\
& -Q(x, y, z),
\end{aligned}
$$

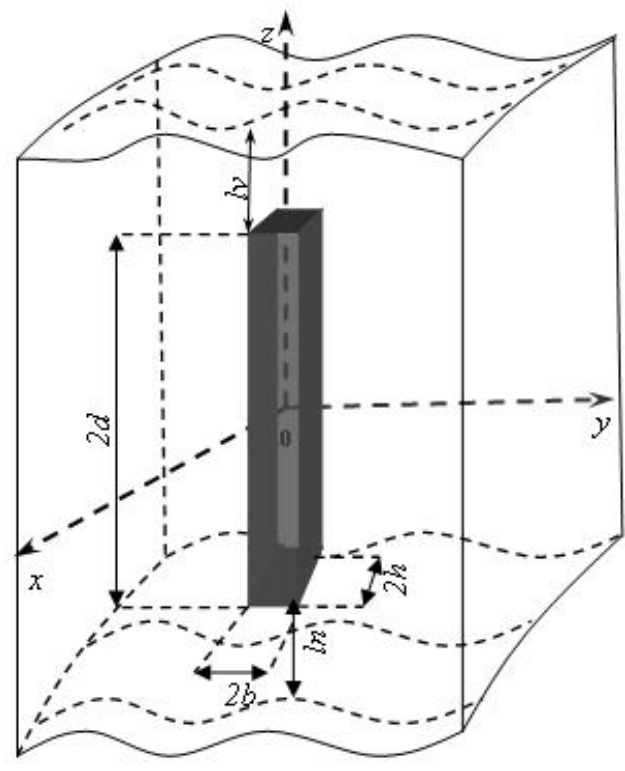

Fig. 1. Isotropic layer with a thin parallelepipedic inclusion. where $\Delta=\frac{\partial^{2}}{\partial x^{2}}+\frac{\partial^{2}}{\partial y^{2}}+\frac{\partial^{2}}{\partial z^{2}}$ is the Laplace operator in Cartesian rectangular coordinate system.

We approximate the function $\theta(0,0, z)$ as (Fig. 2)

$\theta(0,0, z)=\theta_{1}+\sum_{j=1}^{n-1}\left(\theta_{j+1}-\theta_{j}\right) \cdot S_{-}\left(z-z_{j}\right)$

Here, $\left.\quad z_{j} \in\right]-d ; d\left[, z_{1} \leq z_{2} \leq \ldots \leq z_{n-1} ; \theta_{j}(j=\overline{1, n-1})\right.$ are unknown approximating temperature values.

By substituting the expression (7) in equation (6), we obtain:

$\Delta T=\Lambda_{0} \cdot\left\{\left[\theta_{1} \cdot N(z, d)+\sum_{j=1}^{n-1}\left(\theta_{j+1}-\theta_{j}\right) \cdot N\left(z, z_{j}, d\right)\right] \times\right.$

$\times\left[\delta_{x}^{\prime \prime}(x, y)+\delta_{y}^{\prime \prime}(x, y)\right]+$

$\left.+\left[\theta(0,0,-d) \cdot \delta_{-}^{\prime}(z+d)-\theta(0,0, d) \cdot \delta_{+}^{\prime}(z-d)\right] \cdot \delta(x, y)\right\}-$

$-Q(x, y, z)$,

where $N\left(z, z_{j}, d\right)=S_{-}\left(z-z_{j}\right)-S_{+}(z-d)$.

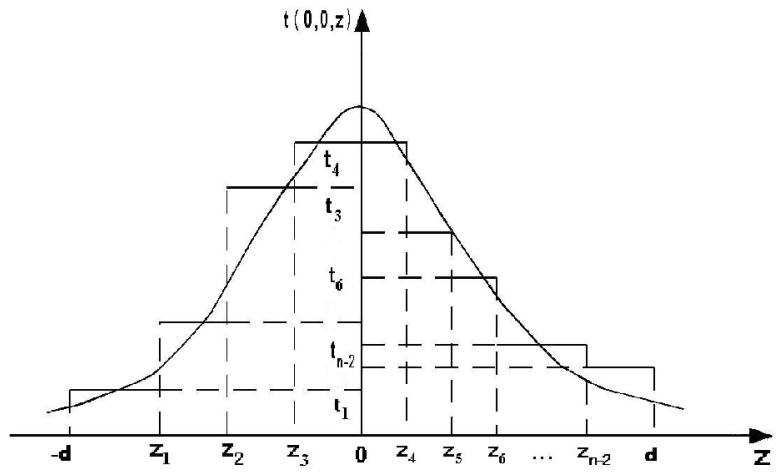

Fig. 2. Approximation of the function $\theta(0,0, z)$. 


\section{Construction of analytical solution to the boundary problem}

Applying the Fourier integral transform by the coordinates $x$ and $y$ to the equation (8) and to the boundary conditions (3) and taking into account the relation (4), we obtain the ordinary differential equation with constant coefficients

$\frac{d^{2} \bar{T}}{d z^{2}}-\gamma^{2} \bar{T}=\frac{\Lambda_{0}}{2 \pi}\left\{\theta(0,0,-d) \cdot \delta_{-}^{\prime}(z+d)-\theta(0,0, d) \times\right.$ $\times \delta_{+}^{\prime}(z-d)-$

$\left.-\gamma^{2} \cdot\left[\theta_{1} \cdot N(z, d)+\sum_{j=1}^{n-1}\left(\theta_{j+1}-\theta_{j}\right) \cdot N\left(z, z_{j}, d\right)\right]\right\}-$

$-\frac{Q_{0}}{2 \pi} \cdot N(z, d)$

and boundary conditions

$\left.\frac{d \bar{T}}{d z}\right|_{z=d+l_{v}}=-\left.\frac{\alpha_{v}}{\lambda_{1}} \bar{T}\right|_{z=d+l_{v}}$

$\left.\frac{d \bar{T}}{d z}\right|_{z=-d-l_{n}}=\left.\frac{\alpha_{n}}{\lambda_{1}} \bar{T}\right|_{z=-d-l_{n}}$.

Here, $\bar{T}=\frac{1}{\sqrt{2 \pi}} \iint_{-\infty}^{\infty} e^{i(\xi x+\eta y)} T d x d y$ is the function $T(x, y, z)$ transformant; $\xi, \eta$ - parameters of the integral Fourier transform; $i=\sqrt{-1}-$ imaginary unit; $\gamma^{2}=\xi^{2}+\eta^{2}$.

The general solution of the equation (9) is:

$\bar{T}=C_{1} e^{\gamma z}+C_{2} e^{-\gamma z}+\frac{1}{2 \pi} \times$

$\times\left\{\Lambda_{0} \cdot\left[\theta(0,0,-d) \cdot \operatorname{ch} \gamma(z+d) \cdot S_{-}(z+d)-\right.\right.$

$-\theta(0,0, d) \cdot \operatorname{ch} \gamma(z-d) \cdot S_{+}(z-d)+\theta_{1} \cdot \Phi(\gamma, z)+$

$\left.\left.+\sum_{j=1}^{n-1}\left(\theta_{j+1}-\theta_{j}\right) \Phi\left(\gamma, z, z_{j}\right)\right]+\frac{Q_{0}}{\gamma^{2}} \Phi(\gamma, z)\right\}$,

where $C_{1}, C_{2}$ are intergrating constants;

$\Phi(\gamma, z)=N(z, d)-\operatorname{ch} \gamma(z+d) S_{-}(z+d)+$

$+\operatorname{ch} \gamma(z-d) S_{+}(z-d)$

$\Phi\left(\gamma, z, z_{j}\right)=N\left(z, z_{j}, d\right)-\operatorname{ch} \gamma\left(z-z_{j}\right) S_{-}\left(z-z_{j}\right)+$

$+\operatorname{ch} \gamma(z-d) S_{+}(z-d)$.

Having applied the boundary conditions (10), a partial solution of the problem (9), (10) is obtained

(9)
$\bar{T}=\frac{1}{2 \pi}\left\{\Lambda_{0} \cdot\left[\theta(0,0, d) \cdot\left(\frac{2 F_{1}(\gamma)}{\Delta_{*}} \cdot F(\gamma, z)-\right.\right.\right.$

$\left.-\operatorname{ch} \gamma(z-d) \cdot S_{+}(z-d)\right)+$

$+\theta(0,0,-d) \cdot\left(\operatorname{ch} \gamma(z+d) \cdot S_{-}(z+d)-\frac{2 F_{2}(\gamma)}{\Delta_{*}} \times\right.$

$\times F(\gamma, z))+\theta_{1} \cdot\left(\Phi(\gamma, z)+\frac{2 F_{3}(\gamma)}{\Delta_{*}} F(\gamma, z)\right)+$

$\left.+\sum_{j=1}^{n-1}\left(\theta_{j+1}-\theta_{j}\right) \cdot\left(\Phi\left(\gamma, z, z_{j}\right)+\frac{2 F_{4}(\gamma)}{\Delta_{*}} \cdot F(\gamma, z)\right)\right]+$

$\left.+\frac{Q_{0}}{\gamma}\left[\frac{\Phi(\gamma, z)}{\gamma}+\frac{2 F_{5}(\gamma)}{\Delta_{*}} F(\gamma, z)\right]\right\}$.

Here,

$$
\begin{aligned}
& \Delta_{*}=\left(\gamma+\frac{\alpha_{n}}{\lambda_{1}}\right) \cdot\left(\gamma+\frac{\alpha_{v}}{\lambda_{1}}\right) \cdot e^{\gamma\left(2 d+l_{v}+l_{n}\right)}- \\
& -\left(\gamma-\frac{\alpha_{n}}{\lambda_{1}}\right) \cdot\left(\gamma-\frac{\alpha_{v}}{\lambda_{1}}\right) \cdot e^{-\gamma\left(2 d+l_{v}+l_{n}\right)} \\
& F_{1}(\gamma)=\gamma \operatorname{sh} \gamma l_{v}+\frac{\alpha_{v}}{\lambda_{1}} \operatorname{ch} \gamma l_{v} ;
\end{aligned}
$$

$F_{2}(\gamma)=\gamma \operatorname{sh} \gamma\left(2 d+l_{v}\right)+\frac{\alpha_{v}}{\lambda_{1}} \operatorname{ch} \gamma\left(2 d+l_{v}\right)$

$F_{3}(\gamma)=\gamma\left(\operatorname{sh} \gamma\left(2 d+l_{v}\right)-\operatorname{sh} \gamma l_{v}\right)+$

$+\frac{\alpha_{v}}{\lambda_{1}}\left(\operatorname{ch} \gamma\left(2 d+l_{v}\right)-\operatorname{ch} \gamma l_{v}\right)$

$F_{4}(\gamma)=\gamma\left(\operatorname{sh} \gamma\left(d+l_{v}-z_{i}\right)-\operatorname{sh} \gamma l_{v}\right)+$

$+\frac{\alpha_{v}}{\lambda_{1}}\left(\operatorname{ch} \gamma\left(d+l_{v}-z_{i}\right)-\operatorname{ch} \gamma l_{v}\right)$

$F_{5}(\gamma)=\frac{F_{3}(\gamma)}{\gamma}$

$F(\gamma, z)=\gamma \operatorname{ch} \gamma\left(z+d+l_{n}\right)+\frac{\alpha_{n}}{\lambda_{1}} \operatorname{sh} \gamma\left(z+d+l_{n}\right)$.

Changing the relation (12) to the original, we obtain the expression for the required temperature

$$
\begin{aligned}
& T=\frac{1}{\pi^{2}} \iint_{0}^{\infty}\left\{\operatorname { c o s } \xi x \operatorname { c o s } \eta y \left[\Lambda_{0}(\theta(0,0, d) \times\right.\right. \\
& \left(\frac{2 F_{1}(\gamma)}{\Delta_{*}} \cdot F(\gamma, z)-\operatorname{ch} \gamma(z-d) \cdot S_{+}(z-d)\right)+
\end{aligned}
$$

$+\theta(0,0,-d) \cdot\left(\operatorname{ch} \gamma(z+d) \cdot S_{-}(z+d)-\frac{2 F_{2}(\gamma)}{\Delta_{*}} F(\gamma, z)\right)+$ 


$$
\begin{aligned}
& +\theta_{1}\left(\Phi(\gamma, z)+\frac{2 F_{3}(\gamma)}{\Delta_{*}} F(\gamma, z)\right)+ \\
& \left.+\sum_{j=1}^{n-1}\left(\theta_{j+1}-\theta_{j}\right) \cdot\left(\Phi\left(\gamma, z, z_{j}\right)+\frac{2 F_{4}(\gamma)}{\Delta_{*}} F(\gamma, z)\right)\right)+ \\
& \left.\left.+\frac{Q_{0}}{\gamma}\left(\frac{\Phi(\gamma, z)}{\gamma}+\frac{2 F_{5}(\gamma)}{\Delta_{*}} F(\gamma, z)\right)\right]\right\} d \xi d \eta .
\end{aligned}
$$

The unknown approximating temperature values $\theta_{i}(i=1,2, \ldots, n)$ and values $\theta(0,0, \pm d)$ can be found by solving the system of $n+2$ linear algebraic equations derived from the expression (13).

Thus, the desired temperature field in nonhomogeneous layer is described by the formula (13) from which we receive the temperature value at an arbitrary point of layer and of foreign inclusion.

\section{Conclusions}

Using generalized functions and piecewise linear approximation of excess temperature $\theta(0,0, z)$ by height of foreign inclusion $z \in]-d ; d[$, with the expression (7), the thermal conductivity equation (8) with a singular right-hand side has been built. Using the Fourier integral transform, the analytical solution (13) of the boundary thermal conductivity problem (1), (3) has been found, which allows in an arbitrary point to calculate the temperature value based on developed new algorithms and software tools and to predict operation modes of individual elements and blocks of microelectronic devices and to identify the unknown parameters as well as increase the heat resistance, which increases their lifetime.

\section{References}

1. A.F. Barvinskyy, V.I. Gavrysh. Nonlinear thermal conductivity problem for nonhomogeneous layer with internal heat sources // Journal of Mechanical Engineering, 2009, Vol. 12, № 1. - P. 47-53. (in Ukrainian).

2. Gavrysh V.I., Fedasyuk D.V. The method for calculation of temperature fields for thermosensitive piecewise homogeneous strip with foreign inclusion // Industrial Heat Engineering. 2010. - Vol. 32, № 5. - P. 18-25. (in Ukrainian).

3. Gavrysh V.I., Fedasyuk D.V., Kosach A.I. Boundary thermal conductivity problem for the layer with cylindrical inclusion // Physicochemical Mechanics of Materials. - 2010. - 46, №5. - P. 115 - 120. (in Ukrainian).

4. V.I. Gavrysh, D.V. Fedasyuk. Thermal simulation of heterogeneous structural components in microelectronic devices // Semiconductor Physics, Quantum Electronics \& Optoelectronics 13, No. 4, p. 439-443 (2010).

5. Ya.M. Podstryhach, V.A. Lomakin, Yu.M. Kolyano, Thermoelasticity of Heterogeneous Body Structure. Nauka. Moscow, 1984 (in Russian).

6. Yu.M. Kolyano, Methods of Heat Conductivity and Thermoelasticity of Heterogeneous Bodies. Naukova Dumka, Kyiv (1992) (in Ukrainian).

7. Korn G., Korn T. Mathematical Handbook for Scientists and Engineers. Moscow: Nauka, 1977. 720 p. (in Russian) 